\title{
Virus-like Particle Vaccine Containing Toxoplasma gondii Rhoptry Protein 13 Induces Protection against T. gondii ME49 Infection in Mice
}

\author{
Hae-Ji Kang1, Ki-Back Chu', Su-Hwa Lee1, Min-Ju Kim¹, Hyunwoo Park², Hui Jin², Fu-Shi Quan,3,4* \\ 'Department of Biomedical Science, Graduate School, Kyung Hee University, Seoul 02447, Korea; '2Health Park Co., Ltd., Seoul 06627, Korea; \\ ${ }^{3}$ Department of Medical Zoology, Kyung Hee University School of Medicine, Seoul 02447, Korea; ${ }^{4}$ Medical Research Center for Bioreaction to \\ Reactive Oxygen Species and Biomedical Science Institute, School of Medicine, Graduate school, Kyung Hee University, Seoul 02447, Korea
}

\begin{abstract}
Toxoplasma gondii can infect humans worldwide, causing serious diseases in pregnant women and immunocompromised individuals. T. gondii rhoptry protein 13 (ROP13) is known as one of the key proteins involved in host cell invasion. In this study, we generated virus-like particles (VLPs) vaccine expressing T. gondii rhoptry ROP13 and investigated VLPs vaccine efficacy in mice. Mice immunized with ROP13 VLPs vaccine elicited significantly higher levels of $T$. gondii-specific $\lg G, \lg G 1, \lg \mathrm{G} 2 \mathrm{a}$, and $\lg A$ antibody responses following boost immunization and challenge infection, whereas antibody inductions were insignificant upon prime immunization. Differing immunization routes resulted in differing antibody induction, as intranasal immunization (IN) induced greater antibody responses than intramuscular immunization (IM) after boost and challenge infection. IN immunization induced significantly higher levels of IgG and IgA antibody responses from feces, antibody-secreting cells (ASCs), $\mathrm{CD} 4^{+} \mathrm{T}, \mathrm{CD} 8^{+} \mathrm{T}$ cells and germinal center $\mathrm{B}$ cell responses in the spleen compared to IM immunization. Compared to IM immunization, IN immunization resulted in significantly reduced cyst counts in the brain as well as lesser body weight loss, which contributed to better protection. All of the mice immunized through either route survived, whereas all nailve control mice perished. These results indicate that the ROP13 VLPs vaccine could be a potential vaccine candidate against $T$. gondii infection.
\end{abstract}

Key words: Toxoplasma gondii, rhoptry protein 13, virus-like particle

Toxoplasma gondii can infect almost all warm-blooded animals such as humans, animals, and birds [1]. Although T. gondii transmission occurs through feline feces, ingestion of raw, undercooked meat contaminated with T. gondii tachyzoites and bradyzoites leads to human infection. Symptoms can be severe in the immunocompromised or in patients receiving organ transplantation, as its infection can affect various organs including the brain and the eyes. Vertical transmission of $T$. gondii through the placenta can occur, and its fetal infection induces abortion and stillbirth in pregnant women [2]. An effective human vaccine against T. gondii infection is currently unavailable. DNA, subunit or protein vaccines against T. gondii have been studied but were unsuccessful [3,4]. As such, finding novel vaccines that are highly effective against $T$. gondii in-

- Received 22 July 2019, revised 23 September 2019, accepted 23 September 2019.

*Corresponding author (quan01@gmail.com)

(c) 2019, Korean Society for Parasitology and Tropical Medicine

This is an Open Access article distributed under the terms of the Creative Commons Attribution Non-Commercial License (http://creativecommons.org/licenses/by-nc/4.0) which permits unrestricted non-commercial use, distribution, and reproduction in any

medium, provided the original work is properly cited. fection remains a great challenge for researchers. Recently, we reported that influenza virus-like particles (VLPs) vaccines containing T. gondii antigenic proteins (IMC, MIC8, ROP18, ROP13, ROP4) provide protection against T. gondii (RH) and/ or T. gondii (ME49) infections [5-8]. T. gondii ROP13 secreted by $T$. gondii rhoptry organ has been recognized as an important antigen. Since there is no comparative study on immunization route on ROP13 VLPs, in this study, the vaccine efficacies induced by ROP13 VLPs immunization by intramuscular (IM) and intranasal (IN) routes were evaluated and compared.

Recombinant baculoviruses expressing ROP13 protein and related influenza VLPs vaccine were generated and characterized as indicated previously [8]. Female BALB/c mice ( $n=6$ per group) were used for ROP13 VLPs immunization by intranasal or intramuscular routes as described previously [8]. Blood from mice was collected by retro-orbital plexus puncture 30 days after prime and boost immunization and challenge infection. Isolated sera from blood were used to determine IgG, IgG1, IgG2a, and IgA by enzyme-linked immunosorbent assay (ELISA). Mice fecal samples were collected 30 days after chal- 
lenge infection. Feces homogenized in PBS were centrifuged at 13,500 RPM, 10 min for supernatant acquisition. The obtained supernatants were used to measure IgG and IgA antibody responses [5]. To measure antibody-secreting cell (ASC) from the spleen of sacrificed mice, isolated splenocytes $\left(1 \times 10^{6}\right.$ cells/well) were cultured for 3 days at $37^{\circ} \mathrm{C}$ with $5 \% \mathrm{CO}_{2}$ in plates coated with $4 \mu \mathrm{g} / \mathrm{ml}$ of $T$. gondii $\mathrm{RH}$ antigen. After 3 days, plates were incubated with IgG and IgA (1:2,000 dilution in PBST) for $1 \mathrm{hr}$ at $37^{\circ} \mathrm{C}$ to measure the level of antibodies being secreted. Additionally, to determine the splenic $\mathrm{CD}_{4}^{+}$, $\mathrm{CD}^{+} \mathrm{T}$ cell, and germinal center $\mathrm{B}$ cell populations, splenocytes $\left(1 \times 10^{6}\right.$ cells) were added to each tube. Surface markers were stained with fluorophore-conjugated antibodies specific to cell phenotypes (CD3e-PE-Cy7, CD4-FITC, CD8-PE, CD45FITC, GL7-PE) (BD Biosciences, San Jose, California, USA). Cells were acquired and data were analyzed using BD Accuri C6 Flow Cytometer and C6 Analysis software, respectively (BD Biosciences). To determine the cysts count after infection, brain tissues were harvested from mice at 1 month post-infection, and homogenized in $400 \mu \mathrm{l}$ of PBS. Homogenized solutions were resuspended in 45\% Percoll and centrifuged for 20 min at $4^{\circ} \mathrm{C}, 12,100 \mathrm{RPM}$. Afterward, cyst layers were carefully
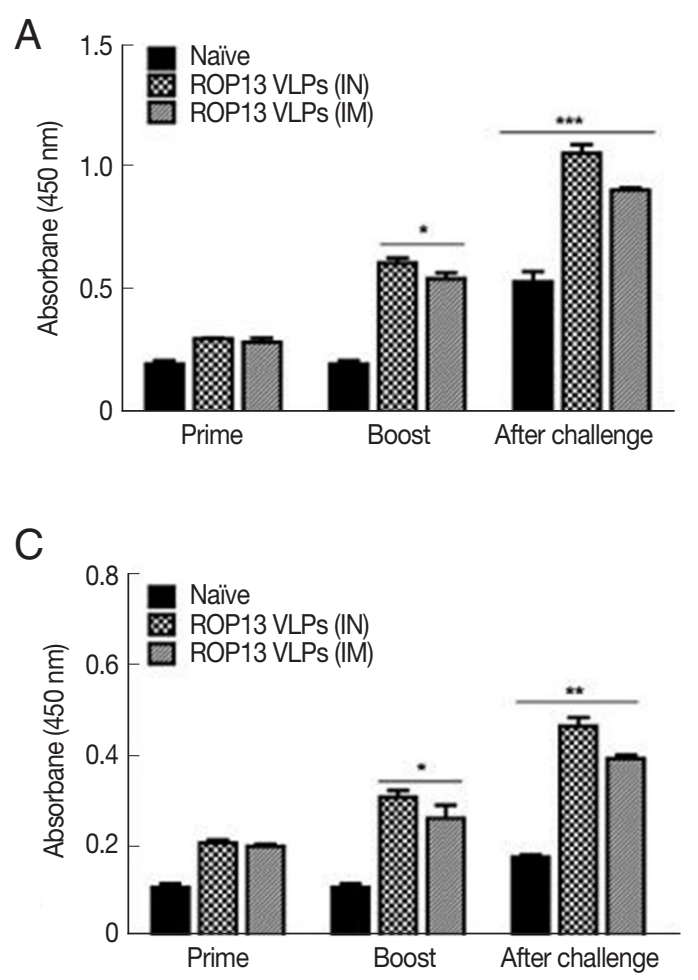

collected and washed with PBS at 6,000 RPM for $20 \mathrm{~min}$. All data were presented as mean \pm SD and statistical significances between groups were analyzed by 1-way ANOVA and Student's $t$-test using GraphPad Prism version 5.0. $P$-values $\left({ }^{*} P<0.05\right.$, $\left.{ }^{* *} P<0.01,{ }^{* *} P<0.001\right)$ were considered statistically significant.

Mice serum samples collected after prime, boost immunization and challenge infection were used to measure T. gondiispecific antibody responses. ROP13 VLPs immunization through the intranasal route (IN) induced higher levels of $T$. gondii-specific IgG (Fig. 1A), IgG1 (Fig. 1B), IgG2a (Fig. 1C) and IgA (Fig. 1D) antibody responses after boost and challenge infection compared to IM. At 4 weeks following challenge infection with $\mathrm{T}$. gondii ME49, CD4 ${ }^{+} \mathrm{T}$ cell, $\mathrm{CD} 8^{+} \mathrm{T}$ cell, and germinal center $\mathrm{B}$ cell responses in immunized mice were observed. As shown in Fig. 2, significantly higher population of $\mathrm{CD}^{+} \mathrm{T}$ cell $(17.8 \%$, Fig. $2 \mathrm{~A}), \mathrm{CD} 8^{+} \mathrm{T}$ cells $(8.7 \%$, Fig. 2B) and germinal center B cells (5.2\%, Fig. 2C) were shown from IN immunization compared to IM immunization. The antibody secretions were measured from splenocytes isolated after challenge infection. Compared to IN immunization, the levels of IgG (Fig. 2D) and IgA (Fig. 2E) in ASC of IM immunization
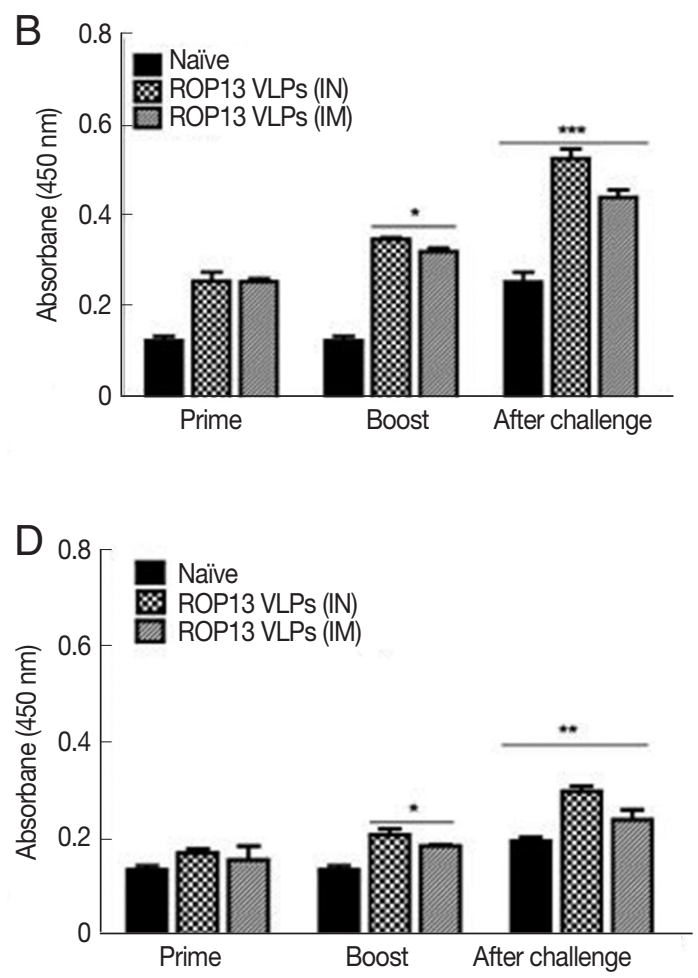

Fig. 1. T. gondii-specific antibody response in sera. (A) T. gondii-specific lgG, (B) IgG1, (C) IgG2a, and (D) IgA in sera were determined. ${ }^{\star} P<0.05,{ }^{* \star} P<0.01,{ }^{* \star *} P<0.001$. Data are expressed as mean $\pm \mathrm{SD}$. 
was lower. These results indicated that IN immunization induced higher levels of ASC responses compared to IM immunization. Based on these results, ROP13 VLPs significantly reduced the cysts count in both IN and IM, with better protection being demonstrated by the former (Fig. 2F). T. gondii-specific IgG and IgA antibody responses in the feces were examined by obtaining feces after challenge infection. As shown in Fig. 3A, B, IN immunization elicited significantly higher levels of T. gondii-specific IgG and IgA antibodies in the feces com- pared to IM immunization. The body weight changes and survival rates were measured for 60 days as illustrated in Fig. 4A, B. IN immunization showed less body weight loss (9\%) compared to IM immunization (13\%). All mice from IN and IM immunization groups survived (Fig. 4B).

In this study, we investigated the protective efficacy of $T$. gondii ROP13 vaccine generated in a virus-like particle form using ROP13, a protein secreted from the rhoptries which assists host cell invasion [4]. In this study, influenza VLPs expressing
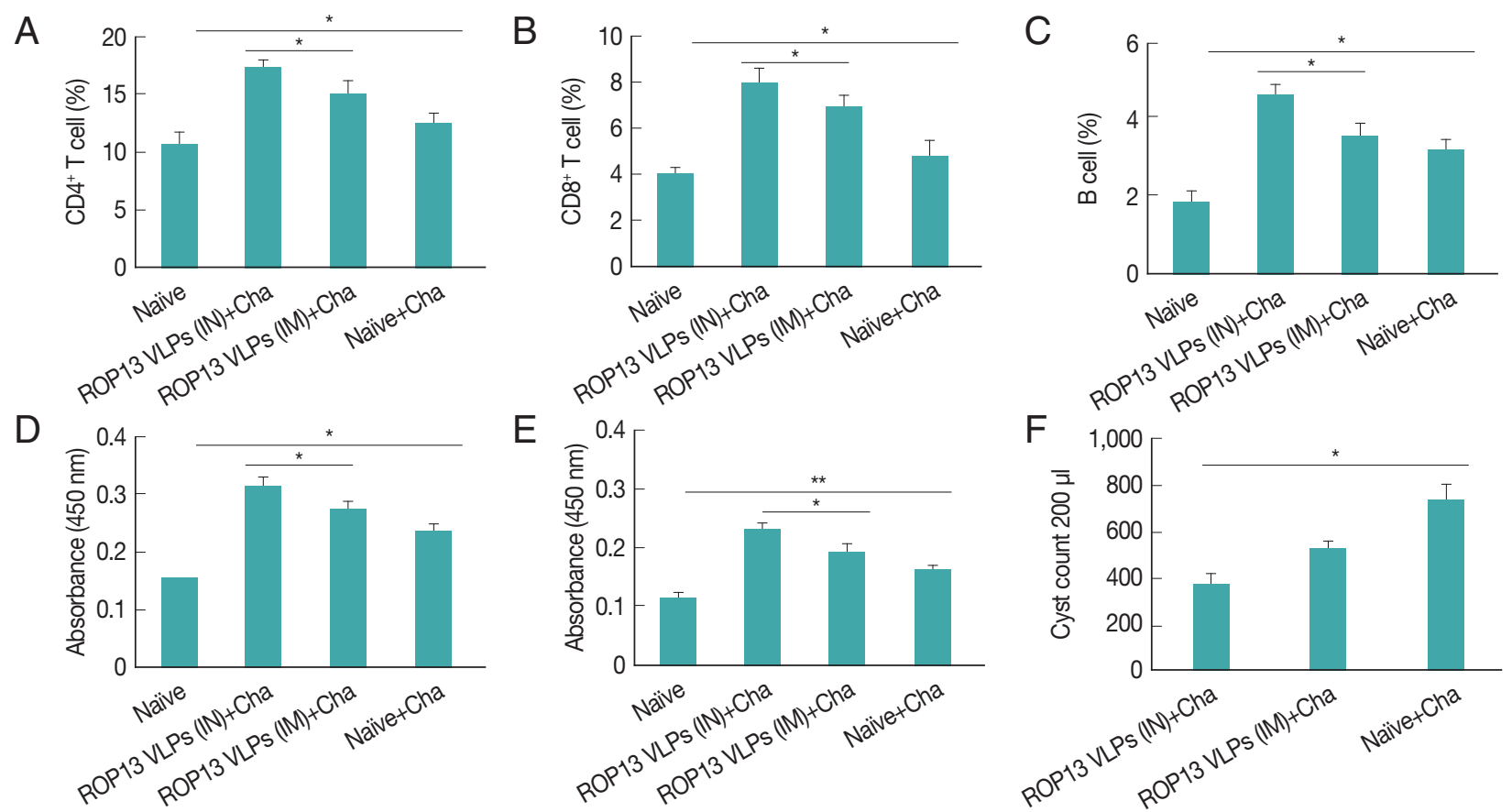

Fig. 2. Protective immune responses induced by ROP13 VLPs vaccination. Four weeks after challenge infection, splenocytes were isolated from mice. (A) CD4 $4^{+}$and (B) CD8 ${ }^{+} \mathrm{T}$ cells in spleen (C) B cell in germinal center. The splenocyte obtained from mice challenge-infected with T. gondii at 1 month after boost. (D) IgG and (E) IgA antibody-secreting responses. (F) Cyst counts in the brain, one month after challenge infection. $\left({ }^{*} P<0.05,{ }^{* *} P<0.01\right)$. Naiive+Cha, Naiive mice were challenge-infected.

A

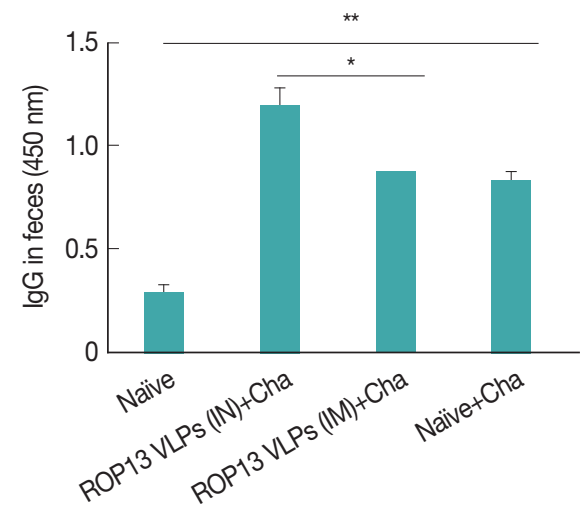

B

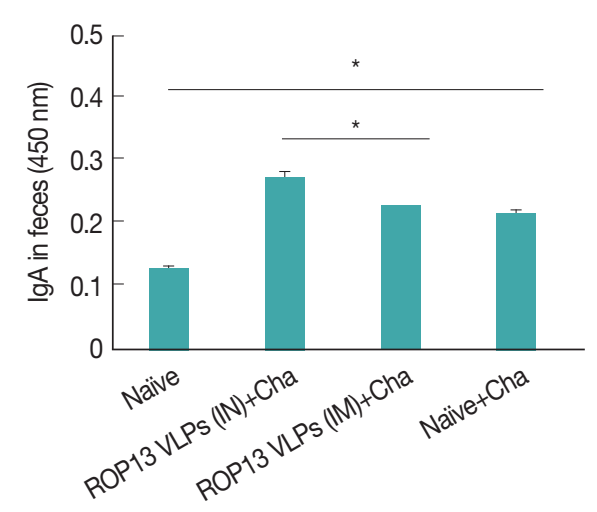

Fig. 3. $\lg G(A)$ and $\lg A(B)$ antibody responses in fecal samples. Feces were collected at 1 month after challenge infection with $T$. gondii. ${ }^{*} P<0.05,{ }^{*} P<0.01$. 

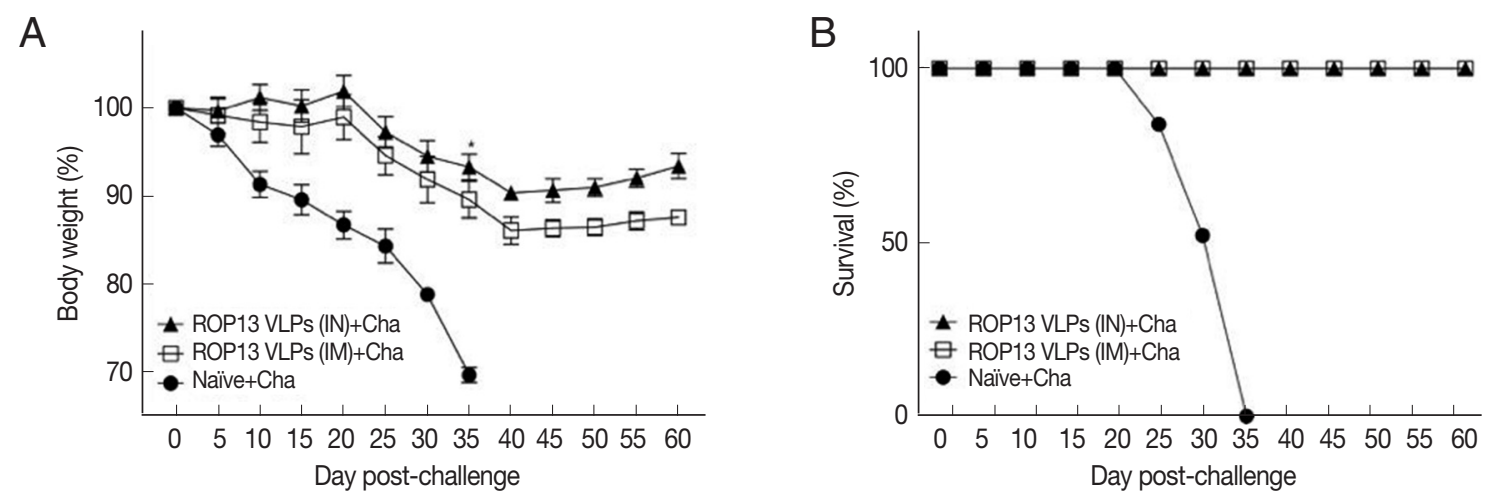

Fig. 4. Body weight (A) and survival (B) of mice immunized with ROP13 VLPs and challenge with T. gondii ME49 (450 cyst). * $P<0.05$, ${ }^{\star *} P<0.01$. Data are expressed as mean \pm SD.

ROP13 were generated and protective immunity induced by IN or IM ROP13 VLPs immunizations were evaluated. In comparison to IM immunization, IN immunization induced higher levels of T. gondii-specific IgG, IgG1, IgG2a, and IgA antibody responses in sera, ASC and T cell responses, along with a significant reduction of cyst counts in the brain.

In general, memory B cells and plasma cells for specific antigens after immunization gradually decrease and are present at low levels. However, upon additional immunization or specific pathogen invasion, rapid proliferation of memory B cells and plasma cells occur $[9,10]$. In the present study, ROP13 VLPs immunization elicited high levels of IgG and IgA secretions from splenocytes after 3 days of in vitro cultures, in which IN immunization showed higher ASC response than IM immunization. We observed significantly higher levels of $\mathrm{CD}^{+}, \mathrm{CD}^{+}{ }^{+} \mathrm{T}$ cell, and B cell responses in VLPs immunization compared to naïve control. CD4 ${ }^{+} \mathrm{T}$ cells activated by ROP13 VLPs differentiated into Th1 and Th2 cells. Subsequent B cell activation by Th1 and Th2 cells leads to IgG2a and IgG1 antibody production in large quantities [11,12]. We found significantly higher levels of IgG, IgG1, IgG2a, and IgA antibody responses in sera from IN than IM immunization after immunization and challenge infection with T. gondii ME49. Mucosal immunity seems to play a critical role in enhancing immunity induced through IN immunization than by IM immunization. In the current study, IN immunization induced higher levels of mucosal IgG and IgA antibody responses in feces compared to IM immunization. Immunized mice were infected with $T$. gondii ME49 through the oral route, which is its natural route of infection. Thus, in the present study, IN immunization induced higher levels of T. gondii-specific IgG and IgA antibody responses in feces which might contribute to better protection against T. gondii ME49 infection than IM immunization. Bradyzoite (cyst) formation in the brains can occur upon infection with T. gondii ME49. ROP13 VLPs immunization by IN route showed significantly decreased brain cyst count. Mice immunized with ROP13 VLPs showed 100\% survival, but all naïve mice died by day 35 after lethal challenge infection.

In summary, this study demonstrates that ROP13 VLPs is an effective vaccine against $T$. gondii ME49 infection in which IN immunization showed higher vaccine efficacy than IM immunization. T. gondii-specific IgG, IgG1, IgG2a, and IgA antibody responses in sera, mucosal IgG and IgA antibodies from feces, $\mathrm{CD}^{+} \mathrm{T}$ cells, and $\mathrm{CD} 8^{+} \mathrm{T}$ cells responses were higher in IN immunization than IM immunization, which may have contributed to significant cyst count reductions in the brain.

\section{ACKNOWLEDGMENTS}

This work was supported by grants from the National Research Foundation of Korea (NRF) (2018R1A2B6003535, 2018 R1A6A1A03025124), the Cooperative Research Program for Agriculture Science \& Technology Development, Rural Development Administration, Republic of Korea (PJ01320501) and from Kyung Hee University in 2019 (KHU-20191220).

\section{CONFLICT OF INTEREST}

The authors declare no conflict of interest related to this study.

\section{REFERENCES}

1. Dubey J, Tiao N, Gebreyes W, Jones J. A review of toxoplasmosis 
in humans and animals in Ethiopia. Epidemiol Infect 2012; 140: 1935-1938.

2. Dubey JP. Toxoplasmosis of Animals and Humans. 2nd ed. Boca Raton, USA. CRC press. 2010.

3. Wang PY, Yuan ZG, Petersen E, Li J, Zhang XX, Li XZ, Li HX, Lv ZC, Cheng T, Ren D, Yang GL, Lin RQ, Zhu XQ. Protective efficacy of a Toxoplasma gondii rhoptry protein 13 plasmid DNA vaccine in mice. Clin.Vaccine Immunol 2012; 19: 1916-1920.

4. Foroutan M, Ghaffarifar F, Sharifi Z, Dalimi A, Jorjani O. Rhoptry antigens as Toxoplasma gondii vaccine target. Clin Exp Vaccine Res 2019; 8: 4-26.

5. Lee SH, Kang HJ, Lee DH, Kang SM, Quan FS. Virus-like particle vaccines expressing Toxoplasma gondii rhoptry protein 18 and microneme protein 8 provide enhanced protection. Vaccine 2018; 36: 5692-5700.

6. Lee SH, Kang HJ, Lee DH, Quan FS. Protective immunity induced by incorporating multiple antigenic proteins of Toxoplasma gondii into influenza virus-like particles. Front Immunol 2018; 9: 3073.
7. Lee SH, Kim AR, Lee DH, Rubino I, Choi HJ, Quan FS. Protection induced by virus-like particles containing Toxoplasma gondii microneme protein 8 against highly virulent RH strain of Toxoplasma gondii infection. PLoS One 2017; 12: e0175644.

8. Kang HJ, Lee SH, Kim MJ, Chu KB, Lee DH, Chopra M, Choi HJ, Park H, Jin H, Quan FS. Influenza virus-like particles presenting both Toxoplasma gondii ROP4 and ROP13 enhance protection against T. gondii Infection. Pharmaceutics 2019; 11: 342.

9. Yoshida T, Mei H, Dörner T, Hiepe F, Radbruch A, Fillatreau S, Hoyer BF. Memory B and memory plasma cells. Immunol Rev 2010; 237: 117-139.

10. McHeyzer-Williams LJ, McHeyzer-Williams MG. Antigen-specific memory B cell development. Annu Rev Immunol 2005; 23: 487-513.

11. Zhu J, Yamane H, Paul WE. Differentiation of effector CD4 T cell populations. Annu Rev Immunol 2009; 28: 445-489.

12. Holdsworth SR, Kitching AR, Tipping PG. Th1 and Th2 T helper cell subsets affect patterns of injury and outcomes in glomerulonephritis. Kidney Int 1999; 55: 1198-1216. 
